5. Булах Е.Г. Прямые и обратные задачи гравиметрии и магнитометрии. Киев: Наук. думка, 2010. 463 с.

6. Гольдшмидт В.И. Оптимизация процесса количественной интерпретации данных гравиразведки. М.: Недра. 1984. 184 с.

7. Тихонов А.Н., Леонов А.С., Ягола А.Г. Нелинейные некорректные задачи. М.: Курс. 2017. 400 с.

8. Ягола А.Г., Ван Янфей, Степанова И.Э., Титаренко В.Н. Обратные задачи и методы их решения. Приложения к геофизике. М.: БИНОМ, Лаборатория знаний. 2014. 216 с.

\title{
РАЗВИТИЕ ВЕКТОРНЫХ МЕТОДОВ ТРАНСФОРМАЦИИ ГЕОПОТЕНЩИАЛЬНЫХ ПОЛЕЙ ПРИ РЕШЕНИИ ОБРАТНЫХ ЗАДАЧ ГРАВИМЕТРИИ
}

\author{
Г.В. Простолупов \\ Горный институт УрО РАН, г. Пермь
}

\begin{abstract}
Аннотация: Рассмотрены методы векторной гравиметрии «Вектор» и «Полюс», разработанные в лаборатории геопотенциальных полей, а также некоторые теоретические аспекты полярного метода, находящегося в состоянии развития. Приведены примеры и сравнение двух методов на моделях геоплотностных разрезов. Оценены их возможности при локализации отрицательных разуплотнений в диапазоне глубин водозащитной и продуктивной толщ ВКМКС и некоторые различия при решении обратной задачи гравиметрии.
\end{abstract}

Ключевые слова: гравиметрия, аномалия, вектор, градиент, трансформация, разуплотнение.

В лаборатории геопотенциальных полей с 1990 гг. ведется развитие методов гравиметрии, заложенных В.М. Новоселицким, основанных на вычислении и трансформации векторов потенциала поля. На первом этапе расчет проводился на калькуляторах, векторы вырисовывались на бумаге и сопоставлялись с геологической обстановкой. С появлением ЭВМ появилась программа «Вектор», которая непрерывно совершенствовалась. Настоящим прорывом стала идея размещения составляющих векторного разложения на вертикальной оси с интерполяцией между горизонтальными плоскостями, и получением 3D куба - позже подобные разложения получили название томографических методов. Подобное было сделано и программно реализовано одними из первых не только в нашей стране, но и в мировой практике, также проработаны теоретическое обоснование, осмысление и вопросы геологической интерпретации трансформант. Первые версии программы «Вектор» были разработаны в операционной системе DOS.

В целом к векторным можно отнести методы, в которых обнаружение искомого аномального объекта (его координаты, размер, избыточная плотность, масса) в той или иной степени связаны с анализом направлений и величин скорейшего изменения потенциала силы тяжести и его производных в пространстве.

С 2000 годов началась адаптация системы «Вектор» к новым требованиям OC Windows. Это привело к полному переустройству внешнего вида программы, еe рабочей области, однако функциональное наполнение сохранилось и усложнилось. Базовые элементы технологии - горизонтальные градиенты силы тяжести - стали вычисляться не только при помощи численных методов и триангуляционной сети, но и аналитически, при помощи истокообразных аппроксимаций. Новый программный модуль «Вектор» позволил обрабатывать геопотенциальные поля (гравитационные, магнитные) с применением векторных технологий, истокообразных аппроксимаций и полярных трансформаций. 
Ряд технических ограничений, как, например, размер сети наблюдений или размер трехмерной диаграммы, оказались сняты в новой версии программы. Это позволило проводить более детальные исследования территории и отказаться от предварительных манипуляций с массивами данных. При этом задействованы многозадачность операционной системы и многопроцессорность оборудования, что позволило комфортно обрабатывать большие объемы данных без ограничений по количеству точек [1].

В системе «Вектор» используется вертикальная производная потенциала $V_{z}$ или вторые производные потенциала - горизонтальные градиенты $V_{z x}, V_{z y}$, первые можно измерять гравиметром, вторые можно вычислить или измерить градиентометром. В 2010 г., как продолжение развиваемого в лаборатории ГП направления векторной гравиметрии, предложен новый метод, названный «Полюс», который задействует геометрические свойства векторов полного градиента потенциала в трехмерном пространстве - сходиться в сторону положительной массы и расходиться от нее в случае отрицательной.

a)

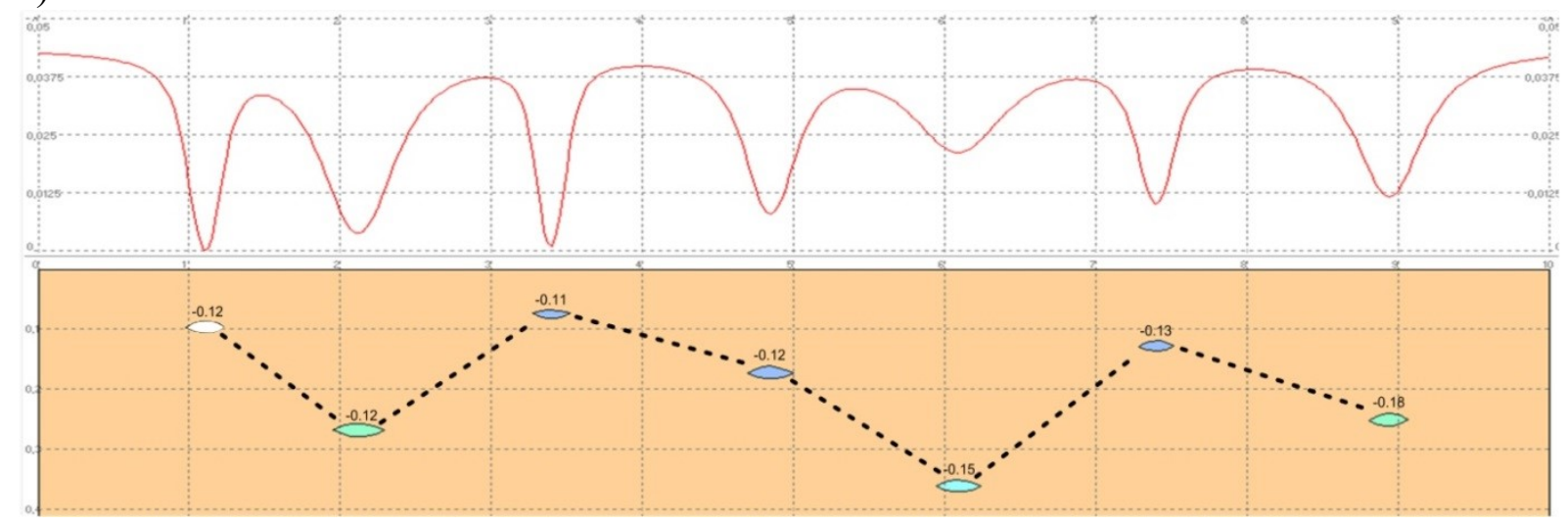

б)

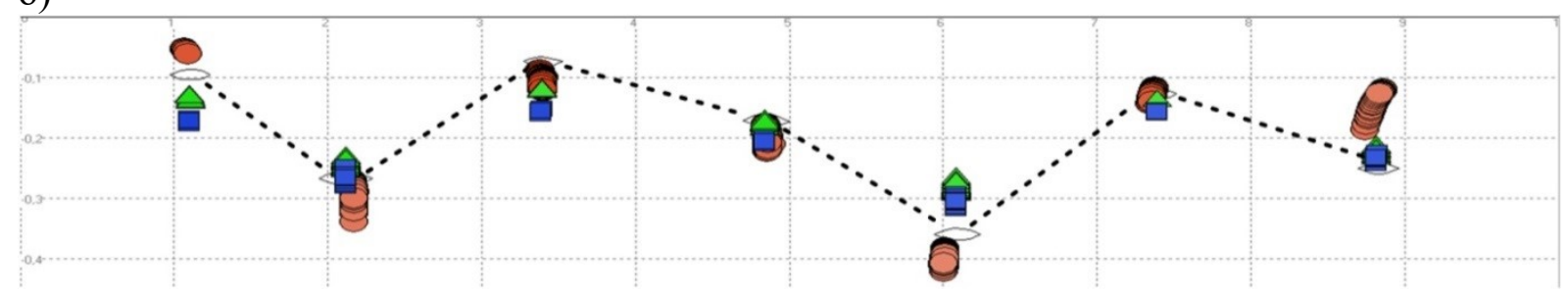

в)

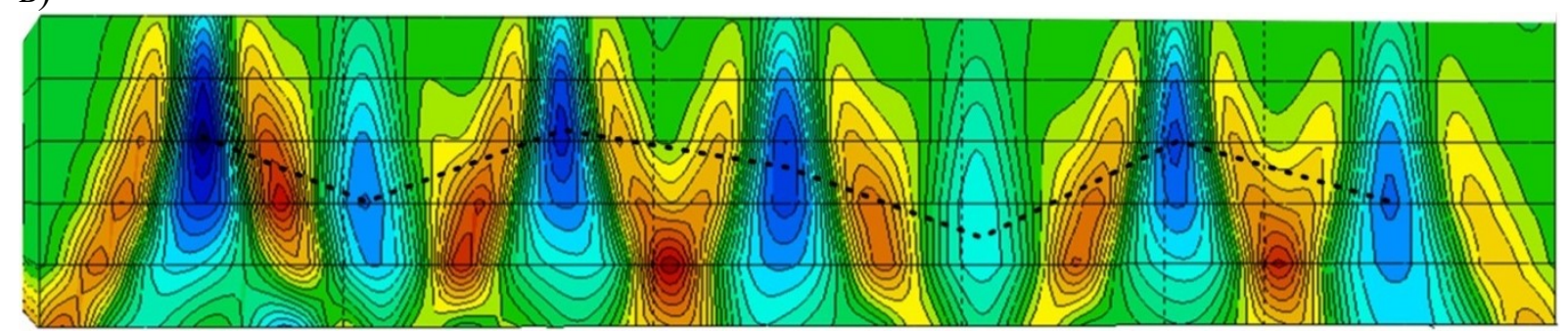

Рис. 1. Модель источников гравитационного поля и график $\Delta \mathrm{g}$ (а), вертикальное сечение трансформанты поля $\Delta \mathrm{g}$, построенное в программе «Полюс» (б) и в системе «Вектор» (в)

Пересечения прямых-продолжений векторов дают точку-полюс, расположенную в области возмущающей массы, $P_{1} P_{2}$ или $P_{3}$, в зависимости от того, поле какой произ- 
водной порождает полюс. Однако при определении полного градиента потенциала $\bar{\nabla} V$ необходимо знать его составляющие по осям координат, т.е. $V_{x} V_{y}$ и $V_{z}$. Значение $V_{z}$ измеряется непосредственно гравиметром на пунктах съемки. Горизонтальные градиенты потенциала $V_{x}, V_{y}$ не измеряются, но их можно вычислить с помощью алгоритма истокообразных аппроксимаций поля [2]. Производные потенциала $V$ вычисляются в следующем порядке. Сначала по имеющейся аппроксимационной конструкции вычисляется потенциал $V$. Затем вычисляются первые горизонтальная $V_{x}$ и вертикальная $V_{z}$ и вторая производная $V_{z x}$. Все эти вычисления можно провести аналитически ввиду относительно слабого их расхождения вблизи нуля аргумента. $V_{z z}$ вычисляется через потенциал $V$ по двумерному аналогу формулы Гендерсона и Зитца, которая может быть получена путем несложных преобразований уравнения Лапласа в конечно-разностном виде. Третья смешанная производная $V_{z z x}$ вычисляется по второй вертикальной, а третья вертикальная производная вычисляется по упомянутой формуле через вертикальную производную $V_{z}[3]$.

В методе «Полюс» добавилась еще одна характеристика - масса аномальных источников. Полюсы образуют скопления в области предполагаемого источника. Каждому такому скоплению точечных источников можно поставить в соответствие объем пространства и аномальную гравитационную массу. При этом, поскольку одна аномалия поля обычно покрывается множеством полигонов и потому порождает целый ряд полюсов близких параметров, за избыточную массу $M$ аномалиеобразующего тела в данном модельном представлении принимается среднее значение масс $m_{i}$ всего множества точек в пределах одного скопления: $M=\sum_{i=1}^{n} m_{i} / n$, где $m_{i}=\left|\vec{g}_{i}\right| \cdot r_{i} / \mathrm{u}$, где $g_{i}$ - полная, восстановленная по компонентам $V_{x}, V_{y}$ и $V_{z}$, сила притяжения $i$-го источника, $r$ - расстояние до него, $G$ - гравитационная постоянная, $n$ - количество полюсов, объединенных в скопление.

Отличие диаграмм скоплений полюсов от диаграмм в системе Вектор заключается в том, что в трехмерном виде изображается схема расположения самих эквивалентных источников с условными характеристиками их размеров и массы, а не интерполированного в трехмерном пространстве поля.

Полюсы разных порядков $P_{I}, P_{I I}$ и $P_{I I I}$ обладают разными свойствами по локализации источников. Исследования на моделях источников показали, что полюсы первых производных $P_{I}$ локализуются с погрешностью или со смещением по горизонтали от источника и в целом имеют меньшую разрешающую способность разделять близкорасположенные тела, чем полюсы $P_{I I}$ и $P_{I I I}$. Однако $P_{I}$ имеет хорошие локализационные свойства при определении глубин изолированных источников [4].

Рассмотрим некоторые характеристики метода «Полюс»и сравнение с системой «Вектор». Гравитационное влияние семи источников, находящихся приблизительно в диапазоне глубин подработанной толщи ВКМКС показано на рис. 1а. Аномальная

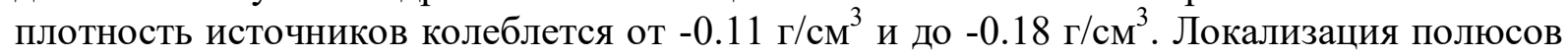
$P_{I}, P_{I I}$ и $P_{I I I}$ на модели семи источников с отрицательной плотностью относительно вмещающих пород показана на рис. 1б. Обнаружен разнонаправленный характер разброса полюсов относительно источников, можно отметить, что свойства локализации полюсов имеют взаимодополняющий характер, в совокупности способствуя более точному определению координат источника поля.

Для сравнения данные модельного поля $\Delta g$ (рис. 1a) обработаны по технологии векторного сканирования. Сечение трехмерной трансформанты приведено на рис. 2в. Можно отметить более обобщенный результат локализации источников, но наблюдает- 
ся хорошо различимая дифференциация по глубинам аномалий в соответствии с расположением источников. Ранее подобный эксперимент был проведен с программой «Вектор» (ДОС), результат был менее выразительным.

Возможность применения программы «Polus2d» при геологической интерпретации детального гравитационного поля рассмотрим на примере схематичного плотностного разреза, имитирующего строение ВКМКС. В карналлитовой зоне, покровной каменной соли и нижней части терригенно-карбонатной толщи разреза смоделированы разуплотнения (рис. 2a). Гравитационный эффект от всей модели можно видеть на графике $V_{z}$. Методом истокообразных аппроксимаций по рассчитанным значениям $V_{z}$ получены величины производных потенциала $V_{z}, V_{x}, V_{y}$, а также высшие производные $V_{z x}, V_{z y}, V_{z z}, V_{z z x}, V_{z z y}, V_{z z z}$. По ним построены градиенты ${ }_{\nabla} V,{ }_{\nabla} V_{z}$, ${ }_{\nabla} V_{z z}$ и рассчитаны координаты пересечений полных векторов в пространстве, в результате чего определены явные отрицательные полюсы $P_{I^{--}}, P_{I^{-}}^{--}$, и $P_{I I I}^{--}$. Полюсы второго $P_{I I}^{--}$и третьего порядков $P_{I I I}^{--}$показали наилучшее совпадение с отрицательными зонами плотностей (рис. 2б).

Поле от данной модели обработано в псевдотрехмерном варианте в системе «Вектор». Источники локализавались с хорошей точностью по горизонтали и менее точно по вертикали. Решение обратной задачи в данном случае можно назвать скорее качественным, чем количественным. Фактором, снижающим точность локализации по вертикали, явилась дискретность диаграммы по оси z и закономерное тяготение локализаций к разностным горизонтальным слоям. В целом не стоит забывать и о вероятности несоответствия глубины залегания пологого горизонтально залегающего источника глубинам локализации аномалий при томографической трансформации поля источника [5].

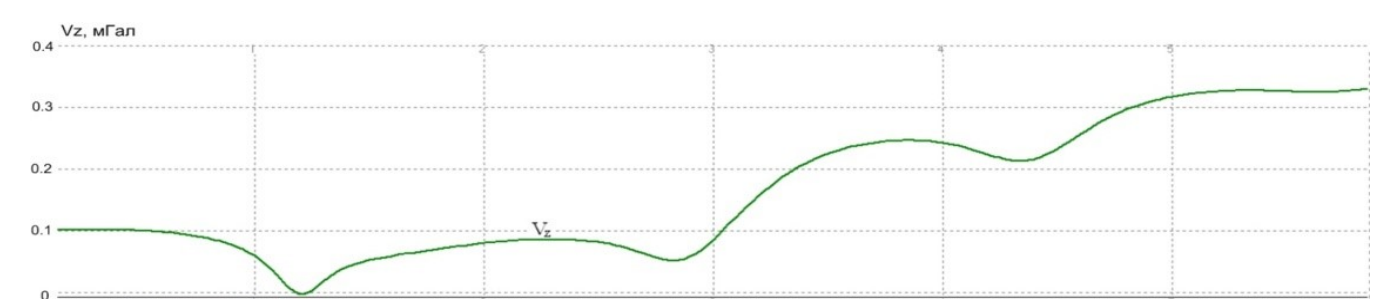

a)

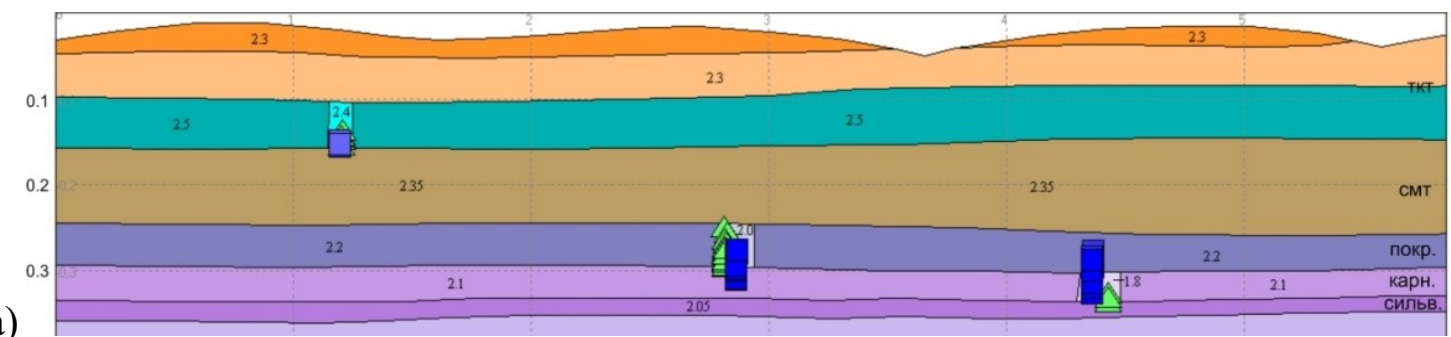

б)

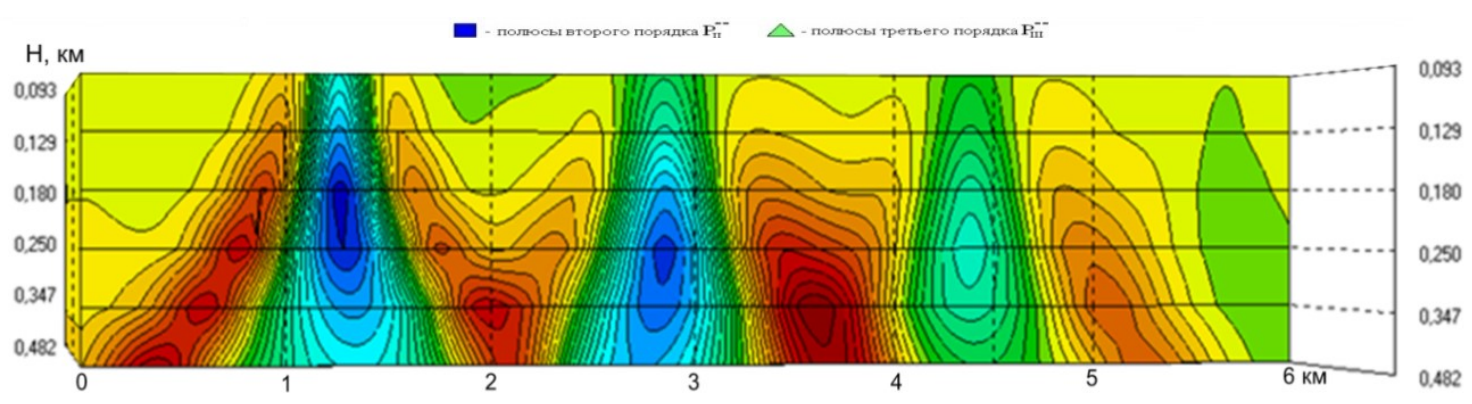

Рис. 2. Модель разреза ВКМКС с разуплотнениями и ее гравитационный эффект $V_{z}$, локализация полюсов $P_{I I} P_{I I I}$ на фоне разреза программе Polus2D (a). Сечение трехмерной диаграммы, построенной по модельному полю, в системе «Вектор» (б) 
Существуют и другие методы трансформации потенциальных полей, относящиеся к томографическим, продвинутые в научном плане системы: «Каскад», «Сигма-3Д», СПАН, методы аппроксимационной томографии и др. Вместе с тем еще в конце прошлого века было отмечено, что «пользователи предпочитают работать с простыми и грубыми методами, но хорошо реализованными в компьютерных системах, а не с совершенными новыми способами, но требующими больших усилий и рутинной работы по подготовке исходного и результативного материала, а также большого времени для вычислений» [6]. Именно к таким методам, по мнению автора, можно отнести систему «Вектор», предоставляющую удобный для восприятия куб интепретационных данных; в результате «информация, считываемая с геоизображений, получаемых в результате гравитационной томографии, может оказаться существенно более содержательной, чем сведения о фиксированном местоположении геоплотностных границ, которые получаются в результате решения обратных задач гравиметрии в заранее выбранных модельных классах источников..» [7].

Перспективы развития метода «Полюс» связаны с расширением использования трансформант высших производных в трехмерном пространстве: версия программы «Polus2D» предполагает получение трансформант первой, второй и третьей производной потенциала; трехмерная «Polus3D» пока располагает только инструментами по работе с первыми производными $V_{z}, V_{x}, V_{y}$.

В настоящее время методы векторной гравиметрии, получившие свое начало еще при возникновении лаборатории геопотенциальных полей, остаются главным инструментом по изучению объемного плотностного строения недр, на основании которого создаются тектонические схемы, восстанавливается история развития территорий, делается прогноз зон нефтегазонакопления, а также потенциально опасных участков разработки ВКМКС [8].

\section{БИБЛИОГРАФИЧЕСКИЙ СПИСОК}

1. Тарантин М.В. Технологическое развитие системы «Вектор» // Стратегия и процессы освоения георесурсов: сб. науч. тр. Вып. 14 / ГИ УрО РАН. - Пермь, 2016. - С. 163-165.

2. Долгаль А.С. Аппроксимация геопотенциальных полей эквивалентными источниками при решении практических задач // Геофизический журнал. - 1999. - Т. 21, № 4. - С. 71-80.

3. Простолупов Г.В., Тарантин М.В. Полярная трансформация производных гравитационного потенциала // Геофизика. - 2013. - № 2. - С. 13-18.

4. Простолупов Г.В. Исследование свойств локализации полюсов относительно источников потенциального поля // Вопросы теории и практики геологической интерпретации геофизических полей: материалы 43-й сес. Междунар. науч. семинара им. Д.Г. Успенского / Воронежский гос. ун-т [и др.]. Воронеж, 2016. - C. 148-150.

5. Бычков С.Г. Современные технологии интерпретации гравиметрических данных при исследованиях на нефть и газ: [Электронный ресурс] / С.Г. Бычков // Нефтегазовое дело. - 2005. - Режим доступа к журн.: http://www.ogbus.ru/ authors/ Bychkov/ Bychkov_1.pdf. - 22 c.

6. Старостенко В.И. О теории и методах интерпретации данных гравиразведки и магниторазведки: некоторые соображения о потребностях сегодняшнего дня // Геофизика и математика: материалы 1-й Всерос. конф. / под ред. В.Н. Страхова; ОИФЗ РАН. - М., 1999. - С. 126-127.

7. Долгаль А.С., Шархимуллин А.Ф. «Гравитационная томография» - практика, опережающая теорию? // Геоінформатика / Украина. - 2009. - № 3. - С. 59-67.

8. Щербинина Г.П. История создания методики «ВЕКТОР» / Г.П. Щербинина // Горное эхо. $-2008 .-$ № 3-4 (33-34). - C. 63-65. 